\title{
Taille de la fratrie et statut social des enfants d'immigrés issus de
} familles nombreuses.

Laure Moguérou, Emmanuelle Santelli, Jean-Luc Primon, Christelle Hamel

\section{Citer ce document / Cite this document :}

Moguérou Laure, Santelli Emmanuelle, Primon Jean-Luc, Hamel Christelle. Taille de la fratrie et statut social des enfants d'immigrés issus de familles nombreuses.. In: Politiques sociales et familiales, n¹11, 2013. pp. 17-30;

doi : 10.3406/caf.2013.2744

http://www.persee.fr/doc/caf_2101-8081_2013_num_111_1_2744

Document généré le 31/05/2016 


\begin{abstract}
The relationship between the number of siblings and social future has rarely been systematically analysed. A study on the subject, published by Dominique Merllié and Olivier Monso in France in Portrait Social - 2007 edition, represents a major contribution to this field. Along the same lines, this article aims to determine if the number of siblings has an effect on the social future of children of immigrants, as well as the nature and extent of this influence. The survey " Trajectoires et Origines" carried out by the French National Institute for Demographic Studies and the French National Institute of Statistics and Economic Studies, between September 2008 and February 2009 , differs from others in that it over-represented this minority sub-population and allowed it to be analysed. The results show that the social discrimination of children from very large families, established for the majority population, is also applicable to the children of immigrants. However, with comparable family and personal characteristics, the differences caused by origin cancel each other out in the male population and are reversed in the female population. The article offers a number of ideas which may explain the differences observed.
\end{abstract}

\title{
Résumé
}

La relation entre la taille de la fratrie et la destinée sociale a rarement fait l'objet d'analyses systématiques. L'étude publiée par Dominique Merllié et Olivier Monso, parue dans France, Portrait Social - Edition 2007, constitue sur ce thème une contribution majeure. Cet article s'inscrit dans son prolongement et vise à déterminer si la taille des fratries affecte le devenir social des enfants d'immigrés, le cas échéant dans quel sens et dans quelle mesure l'enquête Trajectoires et Origines, réalisée conjointement par l'Institut national d'études démographiques et l'Institut national de la statistique et des études économiques entre septembre 2008 et février 2009, a pour particularité d'avoir surreprésenté cette sous-population minoritaire et de permettre une telle analyse. Les résultats montrent que le désavantage social des enfants de familles très nombreuses, établi pour la population majoritaire, vaut également pour les enfants d'immigrés. Mais, à caractéristiques familiales et personnelles comparables, les différences selon l'origine s'annulent dans la population masculine et s'inversent dans la population féminine. Ce faisant, l'article propose quelques pistes explicatives des disparités observées. 


\title{
Taille de la fratrie et statut social des enfants d'immigrés issus de familles nombreuses
}

\author{
\begin{tabular}{c|l} 
& \\
\hline Laure Moguérou & Université Paris-Ouest-Nanterre-Centre de recherche
\end{tabular} \\ Populations et Sociétés (CERPOS) et Institut national \\ d'études démographiques (INED). \\ Emmanuelle Santelli \\ CNRS-Centre Max Weber et Institut national d'études \\ démographiques. \\ Jean-Luc Primon \\ Université Nice Sophia Antipolis- CNRS- Unité de \\ recherches Migrations et Société (URMIS). \\ Christelle Hamel \\ Institut national d'études démographiques. \\ Mots-clés : Fratries - Enfants d'immigrés - Familles nombreuses.
}

La relation entre la taille de la fratrie et la destinée sociale a rarement fait l'objet d'analyses systématiques. L'étude publiée par Dominique Merllié et Olivier Monso parue dans France, Portrait Social Édition 2007 constitue, sur ce thème, une contribution majeure. Cet article s'inscrit dans son prolongement et vise à déterminer si la taille des fratries affecte le devenir social des enfants d'immigrés, le cas échéant dans quel sens et dans quelle mesure. L'enquête Trajectoires et Origines, réalisée conjointement par l'Institut national d'études démographiques et I'Institut national de la statistique et des études économiques entre septembre 2008 et février 2009, a pour particularité d'avoir surreprésenté cette sous-population minoritaire et de permettre une telle analyse. Les résultats montrent que le désavantage social des enfants de familles très nombreuses, établi pour la population majoritaire, vaut également pour les enfants d'immigrés. Mais, à caractéristiques familiales et personnelles comparables, les différences selon l'origine s'annulent dans la population masculine et s'inversent dans la population féminine. Ce faisant, l'article propose quelques pistes explicatives des disparités observées.

es études sociologiques ont montré depuis L longtemps que la position sociale à laquelle accèdent les individus est affectée par de nombreux facteurs. Le milieu social d'origine, notamment le capital scolaire des parents, constitue, en amont du niveau d'éducation des individus, un fort déterminant de la construction des destinées sociales. Le sexe est une autre caractéristique sociale dont I'influence sur la position socioprofessionnelle est désormais bien connue. La relation entre la taille de la fratrie et la destinée sociale au-delà de l'école a été plusieurs fois observée, mais elle a rarement fait l'objet d'analyses systématiques. Après les travaux pionniers de Marcel Bresard (1950) et les analyses de Nicole Tabard dans les années 1980 (1984 et 1986), l'étude publiée par Dominique Merllié et Olivier Monso (2007) à partir des données de l'enquête Formation Qualification Professionnelle [FQP - réalisée par I'Institut national de la statistique et des études économiques (INSEE) en 2003] apporte une contribution majeure. Les résultats montrent que le nombre de frères et de sœurs influence la position sociale des individus en désavantageant ceux issus de familles nombreuses. Ces différences ne s'expliquent pas par un nombre de frères et sœurs plus élevé dans les familles modestes, puisqu'à origine sociale donnée, les différences restent liées à la taille des familles d'origine. De plus, la comparaison avec des données plus anciennes révèle que la liaison ne s'est pas atténuée avec le temps, et qu'elle est plus visible pour les femmes et pour les enfants d'ouvriers et d'employés.

La position sociale varie également selon I'origine migratoire des individus ou de leurs parents. À ce titre, depuis une dizaine d'années, différentes recherches quantitatives (1) se sont intéressées à la question de l'insertion professionnelle des descendants d'immigrés, et ont mis en avant l'existence de discriminations au cours des premières années de la vie active à l'encontre de ceux dont les parents sont venus d'Afrique (méditerranéenne et subsaharienne). Ces difficultés se matérialisent notamment par des retards à l'entrée sur le marché du travail et un surchômage en début de vie active. Cette observation se retrouve dans de nombreuses études, quelle que soit la base de données utilisée. Ainsi, Alain Frickey, Jake Murdoch et Jean-Luc

\footnotetext{
(1) Pendant longtemps, I'information sur l'origine des parents n'était pas collectée dans les grandes enquêtes nationales, et cette invisibilité statistique des descendants d'immigrés interdisait toute étude quantitative sur leur situation professionnelle (Simon, 2008). Dans la dernière décennie, l'introduction de plus en plus fréquente dans les enquêtes publiques de questions sur le pays et la nationalité de naissance des parents a levé cette limite. Les travaux français sur l'insertion économique des migrants et de leurs descendants se sont alors développés rapidement.
} 
Primon (2004) étudient les différences selon l'origine dans les scolarités suivies et l'accès au premier emploi à partir de l'enquête Génération 1998 du Centre d'études et de recherches sur les qualifications (CEREQ) conduite en 2001 et montrent que les jeunes issus de l'immigration maghrébine ont plus de difficultés que les jeunes de la population majoritaire à trouver un premier emploi, même chez les diplômés du supérieur. À partir de la même source, Roxane Silberman et Irène Fournier (2006) mettent en évidence des durées plus longues de chômage après la sortie du système scolaire pour les générations issues du Maghreb, comparativement à ceux issus d'Europe du Sud et au groupe majoritaire. Ces différences selon l'origine des parents dans l'exposition au risque de chômage se retrouvent dans Rachid Bouhmadi et Jean-François Giret (2005) avec des données du CEREQ sur les sortants du système scolaire en 1974, ainsi que dans Romain Aeberhardt et Julien Pouget (2010) avec la base de l'enquête FQP de 2003.

Si I'accès au marché du travail des descendants d'immigrés est bien documenté, les positions socioprofessionnelles qu'ils atteignent une fois en emploi le sont moins. Les travaux des années 1990, comme ceux de Michèle Tribalat (1995) ou de Joëlle Gaymu et Alain Parant (1996) à partir de I'enquête MGIS (Mobilité Géographique et Insertion Sociale) réalisée par I'INSEE et I'Institut d'études démographiques (INED) en 1992, observaient une forte disparité des situations et positions socioprofessionnelles parmi les jeunes issus de I'immigration, et selon le sexe. Dix ans plus tard, Dominique Meurs, Ariane Pailhé et Patrick Simon (2006), à partir de l'enquête Étude de I'histoire familiale (EHF) menée par l'INSEE et l'INED en 1999, ont montré que les descendants d'immigrés occupaient généralement des positions inférieures à celles de la population majoritaire dans la hiérarchie sociale. Plus récente, l'analyse de Mahrez Okba (2012) à partir des données de l'enquête TeO (Trajectoires et Origines) menée conjointement par I'INED et I'INSEE en 2008-2009 confirme la plus grande difficulté d'insertion en emploi stable pour les fils et les filles d'immigrés maghrébins. D'une part, ces derniers mettent deux fois plus de temps pour accéder au premier emploi stable que la population majoritaire ou les filles et fils d'immigrés d'Europe du Sud. D'autre part, le temps passé en emploi stable (période d'emploi d'une durée d'un an ou plus) depuis la sortie du système scolaire est moindre pour les descendants d'immigrés maghrébins, tandis que les périodes de chômage, d'inactivité et d'alternance emploi et chômage sont, pour eux, plus longues et plus fréquentes.
Du côté des emplois occupés, s'il est indéniable que les enfants d'immigrés sont moins souvent ouvriers que leur père, ceux d'origine maghrébine sont tout de même plus nombreux à l'être que ceux d'origine d'Europe du Sud, et plus encore que les hommes de la population majoritaire. II en va de même concernant les emplois d'employés, et on retrouve des écarts analogues auprès des filles. À l'inverse, les filles et les fils d'immigrés maghrébins occupent nettement moins souvent un emploi de profession intermédiaire et de cadre. Ces constats sont confirmés à milieu social comparable (enfants d'ouvriers): les descendants d'immigrés maghrébins sont moins souvent cadres et exercent moins souvent dans les professions intermédiaires, et ils sont davantage ouvriers et employés que les descendants d'immigrés d'Europe du Sud et la population majoritaire. En revanche, les filles d'ouvriers maghrébins sont un peu plus souvent cadres que les filles d'origine ouvrière de la population majoritaire et celles d'origine d'Europe du Sud. L'analyse de M. Okba (2012:6) selon laquelle, à origine sociale ouvrière équivalente, les fils et les filles d'immigrés ont une " destinée » sociale proche de celle des hommes et des femmes de la population majoritaire, ne vaut donc qu'en moyenne.

Dans ces travaux, l'impact de la taille de la fratrie sur la position sociale des enfants d'immigrés a rarement été interrogé. Pourtant, plusieurs études ont fait le constat d'une plus forte fréquence des familles nombreuses parmi les familles immigrées, en particulier dans celles de l'immigration maghrébine (Borrel et Thave, 1989). Pour cette raison, la question du lien entre le nombre de frères et de sœurs et le statut social se pose avec une acuité particulière dans ces familles. Cet article propose une contribution à l'analyse de l'impact du nombre de frères et de sœurs sur la position sociale des enfants à partir du cas des familles maghrébines (très) nombreuses (2). Il s'agit de vérifier si les résultats mis en évidence dans les travaux menés en population générale s'observent également dans la population issue de l'immigration. Les processus qui influent sur la destinée professionnelle des enfants étant à la fois multiples et fortement intriqués, il s'agit de déterminer I'influence respective de différents facteurs expliquant que la position socioprofessionnelle des enfants d'immigrés maghrébins est différente de celle des enfants de la population majoritaire. Après avoir décrit la méthodologie retenue, I'article dressera le portrait des familles (très) nombreuses car ces dernières ne présentent pas les mêmes contours selon qu'elles sont immigrées ou non. Puis il s'agira de déterminer

(2) Sont considérées comme nombreuses les fratries composées de trois enfants et comme très nombreuses celles qui comptent quatre enfants ou plus. 
ce qui d'une origine sociale plus modeste, d'une origine étrangère ou d'une fratrie (très) nombreuse, fait que la position socioprofessionnelle des enfants d'immigrés maghrébins diverge de celle de la population majoritaire. Au final, différentes pistes explicatives seront avancées.

\section{Méthodologie}

Si la méthode adoptée dans cette contribution est en grande partie inspirée du travail de Dominique Merllié et Olivier Monso (2007) réalisé à partir des données de l'enquête Formation et Qualification Professionnelle (FQP) de 2003, certains choix méthodologiques et conceptuels s'en écartent en raison de la spécificité de l'enquête Trajectoires et Origines $(\mathrm{TeO})$ sur laquelle se fonde la présente analyse. Sont ainsi présentés les données mobilisées, le choix des populations sur lesquelles faire porter les analyses et les indicateurs d'origine et de destinée sociales utilisés.

\section{Les données mobilisées}

Réalisée en France métropolitaine, I'enquête TeO est représentative de l'ensemble de la population âgée de 18 ans à 50 ans vivant en ménages ordinaires et résidant en France métropolitaine, mais elle a pour particularité d'avoir surreprésenté les populations minoritaires que sont les immigrés et les descendants d'immigrés. Cela permet d'avoir des effectifs suffisants pour permettre la comparaison statistique entreprise, impossible sur FQP. L'enquête $\mathrm{TeO}$ est multithématique et permet de mettre en relation de nombreuses caractéristiques sociales des individus (dont leurs parcours scolaires et professionnels) avec celles de leur famille d'origine, dont le nombre de frères et de sœurs. Dans I'article de Dominique Merllié et Olivier Monso (2007), la population étudiée est limitée aux «Français de naissance »; elle s'apparente donc à la population majoritaire de l'enquête TeO. D'ailleurs, la taille moyenne des fratries, calculée sur cette sous-population - âgée de 25 ans à 39 ans en 2003 (enquête FQP) et de 30 ans à 44 ans en 2008 (enquête $\mathrm{TeO}$ ) -, est comparable dans les deux enquêtes.

\section{La population d'étude}

Dans le cadre de cet article, sont définies comme « enfants d'immigrés » les personnes nées en France métropolitaine ayant un ou deux parents immigrés (les descendants d'immigrés), mais également les immigrés arrivés en France avant l'âge de 7 ans. L'étude porte sur les personnes âgées de 30 ans à 50 ans. D'une part, dans cette tranche d'âge, $99 \%$ des enquêtés ont terminé leurs études initiales ; $85 \%$ étaient actifs occupés tandis que $93 \%$ des chômeurs et inactifs avaient déjà travaillé par le passé. D'autre part, les effectifs disponibles ne permettent pas de ne retenir que les 40 ans ou plus comme dans l'article de Dominique Merllié et Olivier Monso. En retenant une tranche d'âge aussi large, les individus se trouvent être à des stades différents de leur cycle professionnel, et la position obtenue à la date de l'enquête n'est pas nécessairement définitive. Malgré tout, dans la suite de l'article, le terme de "destinée " est privilégié à celui de " position » car seul le premier permet de considérer la mobilité sociale, en comparaison à une position antérieure ou à une origine donnée. De plus, la situation professionnelle obtenue à l'âge de 30 ans constitue généralement un bon indicateur de la carrière professionnelle à venir, comme en attestent les analyses d'Olivier Monso (2006) et de Manuella Baraton (2006), qui ont montré les difficultés de promotion en cours de carrière.

Alors que les familles nombreuses (trois enfants ou plus) ne représentaient que $10 \%$ des familles en 1999, elles comptent pour $31 \%$ des familles immigrées, $37 \%$ des familles immigrées originaires d'Asie du Sud-Est (Vietnam, Laos, Cambodge), $43 \%$ de celles originaires de Turquie, et respectivement $47 \%$ et $58 \%$ des familles dont la personne de référence est originaire de I'Afrique subsaharienne ou du Maghreb (INSEE, 2005). Dans les courants migratoires européens, la proportion de familles nombreuses avoisine celle des familles non immigrées. Il s'agit ici de se focaliser sur les courants migratoires où les familles (très) nombreuses sont les plus fréquentes. Toutefois, compte tenu de la faiblesse des effectifs dans un certain nombre de migrations non européennes, en raison notamment de la jeunesse de leurs enfants (Beauchemin et al., 2010), n'ont pu être retenus les enfants d'immigrés venus du Sud-Est asiatique, de Turquie ou d'Afrique subsaharienne. L'étude est ainsi limitée aux enfants d'immigrés venus du Maghreb. Au final, l'analyse porte sur deux mille cent cinquante personnes de la population majoritaire et mille trois cent quarante enfants d'immigrés venus du Maghreb.

\section{Indicateurs d'origines et de destinées sociales} Les origines sociales sont présentées à partir d'une classification ad hoc des statuts d'activité et professions du père et de la mère lorsque l'enquêté-e était âgé-e de 15 ans. Dans la plupart des études récentes sur la mobilité sociale, l'origine sociale est approchée par la profession du père. Le choix a été fait ici de prendre en compte l'origine sociale des individus en combinant les groupes socioprofessionnels du père et de la mère, c'est-à-dire en tenant compte à la fois de la position professionnelle occupée par chacun et du statut d'activité des parents. C'est la position la plus élevée qui est retenue. Or, compte tenu du fait que les mères étaient très souvent inactives (du moins pour la population des enfants d'immigrés) et rarement d'un groupe social supérieur à celui du père, elles déterminent 
exceptionnellement un classement différent de celui qu'on aurait obtenu sans cette combinaison. Cette classification permet toutefois de représenter la position de la cellule familiale et de travailler tant sur la mobilité sociale masculine que féminine. Les effectifs des parents dans certaines catégories socioprofessionnelles nécessitent un certain nombre de regroupements. Au final, seront distingués trois types de ménages selon la position socioprofessionnelle combinée des deux parents : les ménages d'indépendants (3); les ménages des classes sociales moyennes et supérieures (les parents dont I'un d'eux occupaient une position de cadre, une profession intellectuelle supérieure ou une profession intermédiaire) ; les ménages des classes sociales populaires (les parents dont l'un d'eux occupaient une position d'employés ou d'ouvriers, qu'ils aient ou non été qualifiés) (tableau 1).

Concernant les positions sociales atteintes par les individus âgés de 30 ans à 50 ans, on conserve une distinction en cinq classes : les indépendants, les cadres et professions intellectuelles supérieures, les professions intermédiaires, les employés et ouvriers qualifiés, les employés et ouvriers non qualifiés. De la même manière, les informations sur le diplôme du père et celles sur le diplôme de la mère ont été combinées pour former une variable unique rendant compte du capital scolaire des parents. Lorsque les deux parents sont diplômés, le cas échéant c'est le diplôme le plus élevé qui est retenu; lorsque l'un des deux diplômes est manquant, nous retenons le diplôme mentionné (tableau 2). Là encore, en raison des faibles effectifs, il a fallu regrouper les modalités "baccalauréat » et " cycle supérieur » dans les analyses qui prennent en compte le diplôme des parents.

\section{Grandir dans une famille immigrée (très) nombreuse : une réalité sociale singulière}

L'enquête TeO permet de croiser les informations relatives à l'origine migratoire et à l'origine sociale des personnes selon le nombre de leurs frères et sœurs. Les résultats confirment que les enfants d'immigrés maghrébins sont plus souvent issus des milieux populaires que ceux de la population majoritaire, et que la fécondité des familles maghrébines est plus élevée que celle de la population majoritaire. Il importe ici de dresser le portrait des familles (très) nombreuses car ces dernières ne présentent pas les mêmes contours selon qu'elles sont immigrées ou non.
Les familles immigrées : des origines sociales populaires et une fécondité élevée

L'observation des catégories socioprofessionnelles des parents immigrés lorsque l'enquêté-e était âgé-e de 15 ans fait apparaître une certaine hétérogénéité, mais aussi une forte concentration dans le bas de I'échelle sociale (tableau 3, p. 22). Près de $80 \%$ des enfants d'immigrés âgés de 30 ans à 50 ans ont des parents appartenant aux milieux populaires (ouvriers et employés qualifiés ou non) contre $47 \%$ des personnes de la population majoritaire. La répartition des parents entre travailleurs non qualifiés et qualifiés se révèle également contrastée: les parents venus d'Afrique du Nord sont bien plus souvent employés et ouvriers non qualifiés (37\%) que ceux de la population majoritaire (12\%). Dans les familles immigrées, I'appartenance aux positions sociales moyennes et supérieures (cadres et assimilés, professions intermédiaires) fait office d'exception ( $9 \%$ ) en comparaison des positions occupées par la population majoritaire $(30 \%)$. En outre, l'inactivité des mères immigrées (57\%) est nettement supérieure à celui des mères de la population majoritaire $(17 \%)$. Le niveau de scolarisation des parents immigrés est également inférieur à celui des parents non immigrés. Près de deux tiers (65\%) des parents venus du Maghreb n'avaient aucun diplôme contre $18 \%$ des parents de la population majoritaire. De plus, $25 \%$ des parents non immigrés étaient titulaires du baccalauréat ou d'un diplôme de l'enseignement supérieur, ce qui n'était le cas que de $8 \%$ des parents venus d'Afrique du Nord. Les enfants d'immigrés maghrébins ont eu, en moyenne, plus de frères et de sœurs $(5,1)$ que les enfants de familles non immigrées $(2,4)$. Ainsi, $80 \%$ d'entre eux ont grandi dans des familles comptant quatre enfants ou plus et $40 \%$ dans des familles de six enfants ou plus, contre respectivement $36 \%$ et $7 \%$ de la population majoritaire.

\section{Profil des familles (très) nombreuses}

Être issu d'une famille (très) nombreuse est aujour$d^{\prime}$ hui comme hier étroitement associé à des origines sociales populaires (Borrel et Thave, 1989), et cela est d'autant plus vrai que les parents sont immigrés (tableau 3). Les enfants d'immigrés appartenant à des familles très nombreuses font partie des ménages populaires dans $85 \%$ des cas, contre $50 \%$ quand ils font partie de familles de taille réduite. Dans le cas de la population majoritaire, les écarts sont un peu moindres mais, surtout, ils n'atteignent pas un tel niveau : respectivement $61 \%$ et $37 \%$. Dans la population majoritaire, les enfants issus de familles très nombreuses appartenant aux classes moyennes et supérieures et aux ménages d'indépendants atteignent chacun près de $20 \%$. Ainsi, ces familles très nombreuses ont des profils bien différents : tandis que les familles d'origine

(3) Moins homogènes socialement, les groupes d'indépendants peuvent difficilement être agrégés aux autres groupes. 


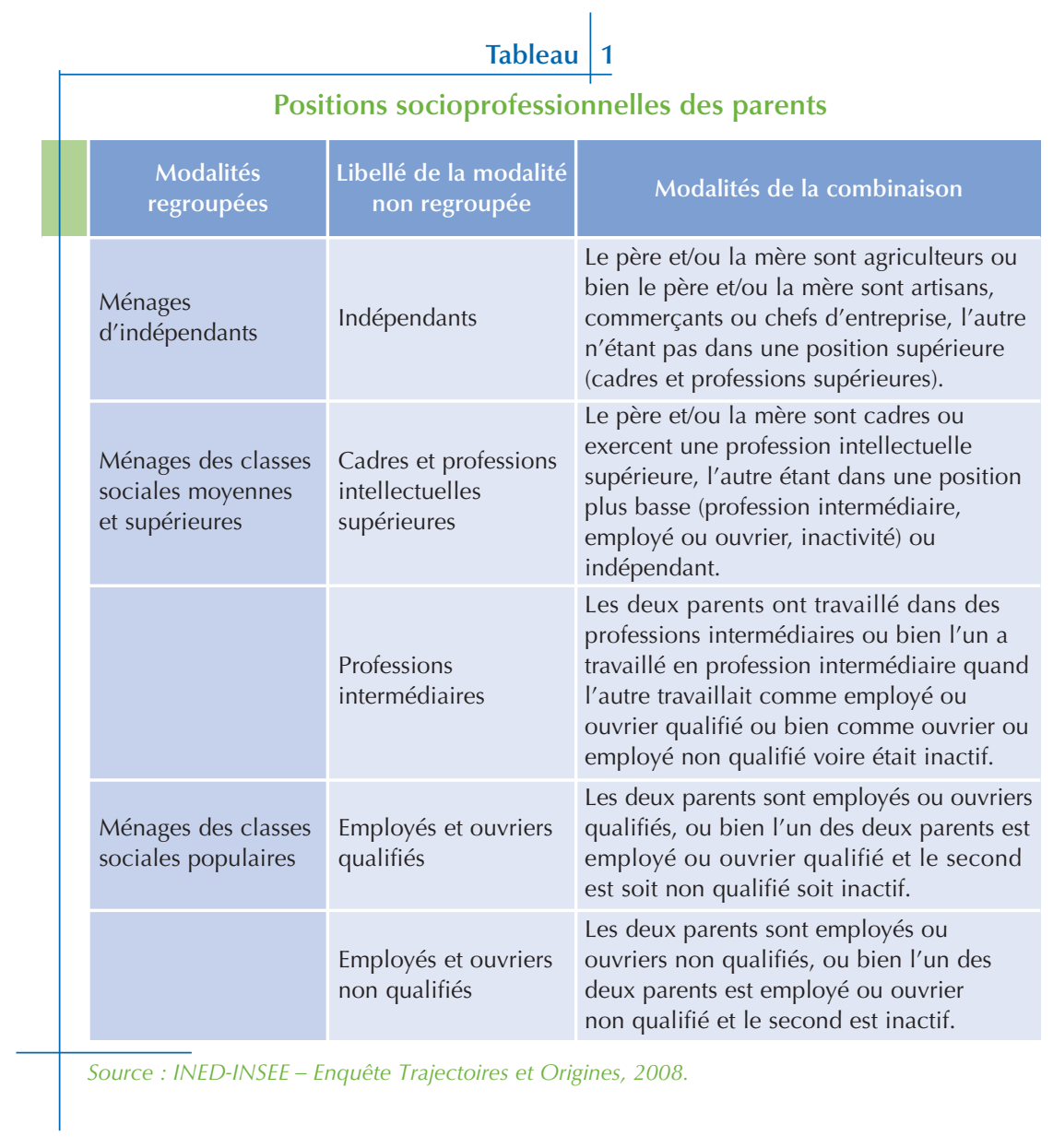

majoritaire contre les deux tiers parmi les enfants d'immigrés. Certes, dans les familles très nombreuses maghrébines, les revenus sont limités du fait que les familles disposent le plus souvent d'un seul salaire (celui du père qui est le plus souvent ouvrier), mais la quête d'ascension sociale consubstantielle au projet migratoire encouragerait une plus grande réussite scolaire et sociale (Santelli, 2001) (4).

\section{Destinée sociale et taille des fratries}

La destinée sociale varie selon l'origine et le sexe... Occuper une profession de cadre (ou assimilé) concerne $17 \%$ des individus de la population majoritaire (19\% des hommes et $15 \%$ des femmes), mais $11 \%$ des filles et fils d'immigrés maghrébins.

maghrébine font très majoritairement partie des classes populaires, dans la population majoritaire, ces familles sont polarisées socialement. Schématiquement, elles se caractérisent soit par leur appartenance à un milieu traditionnel privilégié, soit par une forte vulnérabilité sociale. Le niveau de diplôme témoigne également de ces écarts : les enfants d'immigrés appartenant à des familles très nombreuses ont, dans les trois quarts des cas, des parents qui n'ont obtenu aucun diplôme, tandis que ce n'est le cas que pour $29 \%$ dans la population majoritaire. Et parmi cette dernière, seulement $14 \%$ des parents sont diplômés du supérieur. La part des mères n'ayant jamais travaillé est d'un quart dans la population
Parallèlement, moins de la moitié (42\%) des hommes de la population majoritaire sont employés ou ouvriers (dont $13 \%$ de non qualifiés), contre $58 \%$ des fils d'immigrés venus d'Afrique du Nord

Tableau 2

Niveau de diplôme des parents

\begin{tabular}{|c|c|}
\hline $\begin{array}{l}\text { Libellé de } \\
\text { la modalité }\end{array}$ & Modalités de la combinaison \\
\hline Aucun diplôme & Les deux parents sont non diplômés. \\
\hline CEP-BEPC & $\begin{array}{l}\text { Un des deux parents au moins est titulaire du CEP ou d'un BEPC, } \\
\text { l'autre ayant un diplôme équivalent ou inférieur. }\end{array}$ \\
\hline CAP-BEP & $\begin{array}{l}\text { Un des deux parents au moins est titulaire d'un CAP ou d'un BEP, } \\
\text { l'autre ayant un diplôme équivalent ou inférieur. }\end{array}$ \\
\hline Baccalauréat & $\begin{array}{l}\text { Un des deux parents au moins est titulaire du baccalauréat, l'autre } \\
\text { ayant un diplôme équivalent ou inférieur. }\end{array}$ \\
\hline Supérieur & $\begin{array}{l}\text { Un des deux parents au moins est titulaire d'un diplôme de } \\
\text { l'enseignement supérieur, l'autre ayant un diplôme équivalent } \\
\text { ou inférieur. }\end{array}$ \\
\hline $\begin{array}{l}\text { Cource : INED-INS } \\
\text { deverenu Diplôme } \\
\text { d'études profession }\end{array}$ & $\begin{array}{l}\text { nquête Trajectoires et Origines, } 2008 \text {. } \\
\text { primaires; BEPC : brevet d'études du premier cycle du second degré } \\
\text { al du brevet) ; CAP : certificat d'aptitudes professionnelles ; BEP : brevet } \\
\text { s. }\end{array}$ \\
\hline
\end{tabular}

(4) Cela expliquerait qu'à catégories socioprofessionnelles du père équivalentes (en l'occurrence en comparant la situation quand le père est ouvrier), les enfants d'immigrés obtiennent des résultats comparables de réussite au baccalauréat (Brinbaum et Kieffer, 2005). 
Tableau 3

Répartition par origine sociale et niveau de diplômes des parents selon la taille de la famille (en \%)

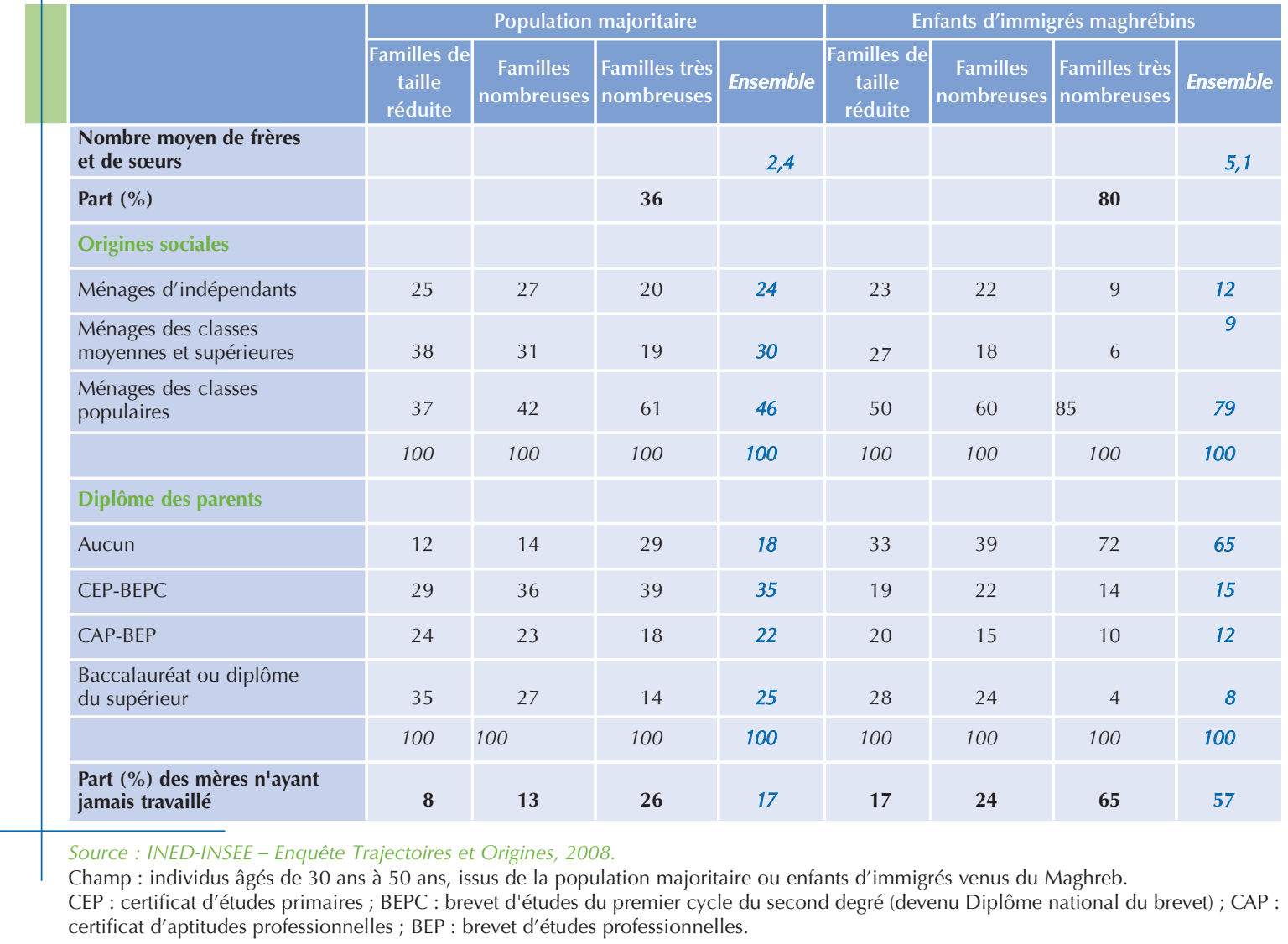

(dont $19 \%$ de non qualifiés). Dans la population féminine, les écarts sont également importants: $55 \%$ des femmes de la population majoritaire et $66 \%$ de celles issues de l'immigration occupent des positions subalternes, et respectivement $28 \%$ et $36 \%$ d'entre elles occupent des emplois non qualifiés (tableau 4 , colonnes «Ensemble »).

\section{... et selon la taille de la fratrie}

Dans les deux populations, I'augmentation de la taille de la fratrie diminue ainsi la probabilité de devenir cadre et augmente celle d'occuper des emplois d'employé-e-s ou d'ouvrier-ère-s non qualifié-e-s. L'effet de la taille de la famille est encore plus préjudiciable aux femmes: dans la population majoritaire, elles sont $15 \%$ à occuper une position de cadre, mais seulement $8 \%$ quand elles sont issues de familles très nombreuses, tandis que pour les hommes l'écart se situe entre $19 \%$ et $13 \%$; parmi les descendants d'immigrés, les femmes sont $15 \%$ à occuper une position de cadre, mais seulement $7 \%$ quand elles sont issues de familles très nombreuses, tandis que pour les hommes l'écart se situe entre $10 \%$ et $8 \%$. Les enfants d'immigrés maghrébins paraissent, d'une part, être moins désavantagés que les enfants de la population majoritaire par le fait d'appartenir à une famille très nombreuse plutôt qu'à une famille de taille réduite, même si la seconde perspective est, pour tous, plus avantageuse pour obtenir une position sociale plus élevée. La différence entre filles et fils d'immigrés dans l'accès aux positions sociales les plus élevées selon la taille de la fratrie est très faible; elle est plus forte au bas de la hiérarchie professionnelle.

\section{Les écarts se maintiennent dans les milieux populaires}

Les tendances mises en évidence supra se confirment pour la sous-population des enfants de milieux populaires (tableau 5, p. 24) : les enfants d'immigrés s'en sortent en moyenne moins bien que population majoritaire (ils sont moins souvent cadres et plus souvent employés ou ouvriers). Et l'effet de la taille de la fratrie se fait ressentir sur les deux populations. Toutefois, il est davantage marqué pour les femmes que pour les hommes, mais il est surtout clairement plus fort dans la population majoritaire que dans la population des enfants d'immigrés. Les femmes d'origine populaire au sein de la population majoritaire et appartenant à des fratries réduites sont $9 \%$ à être devenues 
cadres ou assimilées, mais elles ne sont plus que $3 \%$ quand elles appartiennent à des familles très nombreuses ( $\mathrm{C}^{\prime}$ est le cas pour respectivement $13 \%$ et $8 \%$ des hommes dans la même situation). Du côté des filles d'immigrés maghrébins, elles sont $18 \%$ à être devenues cadres en appartenant à une fratrie réduite (donc le double par rapport aux femmes d'origine populaire).

\section{Démêler l'influence respective \\ des facteurs en jeu}

Les processus qui influent sur la destinée scolaire et professionnelle des enfants sont à la fois multiples et fortement intriqués. Les enfants d'immigrés ont grandi dans un environnement familial qui apparaît moins favorable à leur réussite scolaire : moindre certification des parents, origine sociale populaire et ouvrière plus fréquente, forte inactivité des mères, familles plus souvent nombreuses. Et de fait, environ un quart $(22 \%)$ des enfants d'immigrés maghrébins ont quitté le système scolaire sans diplôme ou avec des diplômes faiblement reconnus sur le marché du travail (tels que le certificat d'études primaires (CEP)), contre $11 \%$ des individus de la population majoritaire. Parallèlement, la part des diplômés du supérieur est de $25 \%$ chez les fils et les filles d'immigrés maghrébins, ce qui est plus faible que dans la population majoritaire (35\%). Or, la force du lien entre le diplôme et l'emploi en France fait du parcours scolaire un enjeu de long terme (Meron et Minni, 1995).

Au final, entre une origine étrangère, une origine sociale plus modeste, une moindre certification scolaire et une fratrie plus nombreuse en moyenne, qu'est-ce qui « explique » que la position socioprofessionnelle des enfants d'immigrés est différente de celle des enfants de la population majoritaire? Pour répondre à cette question, des régressions logistiques ont été élaborées afin d'identifier dans quelle mesure le nombre de frères et de sœurs, d'une part, et le capital scolaire des parents, le milieu social ainsi que les origines migratoires, d'autre part, sont associés au fait d'être devenu-e cadre plutôt que d'occuper une position sociale moindre (tableau 6, p. 25). Cette démarche a ensuite été reproduite mais en explorant le fait d'être devenu-e ouvrier-ère ou employé-e non qualifié-e plutôt que d'avoir atteint une position sociale plus élevée (tableau 7, p. 27). On examine ainsi, à l'inverse, le fait de s'être maintenu dans une position sociale peu favorable, situation courante chez les enfants d'immigrés venus du Maghreb. Dans les deux cas, deux étapes ont été entreprises. Le premier modèle analyse les corrélations entre la position sociale atteinte, l'origine migratoire, l'origine sociale, les

Tableau 4

Catégories socioprofessionnelles de la population majoritaire et des enfants d'immigrés selon le sexe et la taille de la fratrie (en \%)

\begin{tabular}{|c|c|c|c|c|c|c|c|c|}
\hline & \multicolumn{4}{|c|}{ Population majoritaire } & \multicolumn{4}{|c|}{ Enfants d'immigrés maghrébins } \\
\hline & $\begin{array}{c}\text { Familles de } \\
\text { taille } \\
\text { réduite }\end{array}$ & $\begin{array}{c}\text { Familles } \\
\text { nombreuses }\end{array}$ & $\begin{array}{l}\text { Familles très } \\
\text { nombreuses }\end{array}$ & Ensemble & $\begin{array}{c}\text { Familles de } \\
\text { taille } \\
\text { réduite }\end{array}$ & $\begin{array}{c}\text { Familles } \\
\text { nombreuses }\end{array}$ & $\begin{array}{l}\text { Familles très } \\
\text { nombreuses }\end{array}$ & Ensemble \\
\hline \multicolumn{9}{|l|}{ Destinée sociale des hommes } \\
\hline Indépendants & 10 & 13 & 11 & 11 & 18 & 16 & 9 & 10 \\
\hline Professions intermédiaires & 29 & 27 & 24 & 27 & 19 & 20 & 22 & 21 \\
\hline Employés ou ouvriers qualifiés & 26 & 29 & 35 & 29 & 34 & 31 & 40 & 39 \\
\hline $\begin{array}{l}\text { Employés ou ouvriers non } \\
\text { qualifiés }\end{array}$ & 11 & 10 & 17 & 14 & 16 & 6 & 21 & 20 \\
\hline Indépendantes & 5 & 8 & 6 & 6 & 4 & 6 & 3 & 3 \\
\hline Cadres ou assimilées & 21 & 16 & 7 & 15 & 30 & 21 & 7 & 11 \\
\hline Professions intermédiaires & 29 & 23 & 20 & 24 & 23 & 19 & 19 & 20 \\
\hline $\begin{array}{l}\text { Employées ou ouvrières } \\
\text { qualifiées }\end{array}$ & 26 & 25 & 29 & 27 & 20 & 28 & 32 & 30 \\
\hline \multirow[t]{2}{*}{$\begin{array}{l}\text { Employées ou ouvrières } \\
\text { non qualifiées }\end{array}$} & 19 & 28 & 38 & 28 & 23 & 26 & 39 & 36 \\
\hline & 100 & 100 & 100 & 100 & 100 & 100 & 100 & 100 \\
\hline
\end{tabular}




\begin{tabular}{|c|c|c|c|c|c|c|c|c|}
\hline \multicolumn{9}{|c|}{ Tableau 5} \\
\hline \multicolumn{9}{|c|}{$\begin{array}{l}\text { Destinée professionnelle de la population majoritaire et des enfants d’immigrés } \\
\text { de ménages populaires selon le sexe et la taille de la fratrie (en \%) }\end{array}$} \\
\hline & \multicolumn{4}{|c|}{ Population majoritaire } & \multicolumn{4}{|c|}{ Enfants d'immigrés maghrébins } \\
\hline & $\begin{array}{l}\text { Familles de } \\
\text { taille } \\
\text { réduite }\end{array}$ & $\begin{array}{c}\text { Familles } \\
\text { nombreuses }\end{array}$ & $\begin{array}{l}\text { Familles très } \\
\text { nombreuses }\end{array}$ & Ensemble & $\begin{array}{c}\text { Familles de } \\
\text { taille } \\
\text { réduite }\end{array}$ & \begin{tabular}{c|} 
Familles \\
nombreuses
\end{tabular} & $\begin{array}{l}\text { Familles très } \\
\text { nombreuses }\end{array}$ & Ensemble \\
\hline \multicolumn{9}{|l|}{ Destinée sociale des hommes } \\
\hline Indépendants & 8 & 6 & 7 & 7 & 12 & 18 & 8 & 9 \\
\hline Cadres ou assimilés & 13 & 16 & 8 & 12 & 17 & 8 & 7 & 8 \\
\hline Professions intermédiaires & 26 & 16 & 22 & 22 & 19 & 22 & 20 & 20 \\
\hline Employés ou ouvriers qualifiés & 39 & 52 & 40 & 42 & 27 & 41 & 43 & 42 \\
\hline \multirow[t]{2}{*}{$\begin{array}{l}\text { Employés ou ouvriers non } \\
\text { qualifiés }\end{array}$} & 14 & 10 & 23 & 17 & 25 & 11 & 22 & 21 \\
\hline & 100 & 100 & 100 & 100 & 100 & 100 & 100 & 100 \\
\hline \multicolumn{9}{|l|}{ Destinée sociale des femmes } \\
\hline Indépendantes & 4 & 4 & 6 & 5 & 0 & 10 & 3 & 3 \\
\hline Cadres ou assimilées & 9 & 7 & 3 & 6 & 18 & 14 & 7 & 8 \\
\hline Professions intermédiaires & 25 & 20 & 18 & 20 & 16 & 15 & 19 & 19 \\
\hline $\begin{array}{l}\text { Employées ou ouvrières } \\
\text { qualifiées }\end{array}$ & 32 & 32 & 28 & 30 & 32 & 31 & 33 & 33 \\
\hline \multirow[t]{2}{*}{$\begin{array}{l}\text { Employées ou ouvrières } \\
\text { non qualifiées }\end{array}$} & 30 & 37 & 45 & 39 & 34 & 30 & 38 & 37 \\
\hline & 100 & 100 & 100 & 100 & 100 & 100 & 100 & 100 \\
\hline
\end{tabular}

Source: INED-INSEE - Enquête Trajectoires et Origines, 2008.

Champ : individus âgés de 30 ans à 50 ans, issus de la population majoritaire ou enfants d'immigres venus du Maghreb, de ménages populaires

diplômes des parents et le nombre de frères et de sœurs. Dans un second modèle, est ajouté le diplôme de l'enquêté-e. Les analyses ont été conduites séparément pour les hommes et les femmes de façon à explorer si, et dans quelle mesure, les rapports sociaux de sexe modulent les effets de la taille de la fratrie et des origines sociales.

Dans la population masculine, les chances d'accéder au statut de cadre sont plus faibles pour les fils d'immigrés que pour les hommes de la population majoritaire. Les enfants d'immigrés, en particulier quand ils sont issus de familles très nombreuses, ont plus souvent grandi dans des milieux populaires tandis que, parallèlement, les très grandes familles font, pour la plupart d'entre elles, partie des classes populaires. Le premier modèle montre que l'effet de l'origine migratoire disparaît avec la prise en compte des héritages sociaux et scolaires, tandis que l'effet du nombre élevé de frères et de sœurs demeure un désavantage, et ce que les parents soient immigrés ou non (tableau 6, modèle 1). Les effets de reproduction sociale sont très prononcés: la probabilité d'être soi-même cadre plutôt que d'occuper une position inférieure lorsque les parents occupaient des positions de cadres ou de professions intermédiaires est multipliée par deux comparativement à la probabilité des fils d'employés et ouvriers (5). Avoir au moins un parent titulaire du baccalauréat ou diplômé du supérieur est également un facteur étroitement associé au fait d'être soi-même dans une position de cadre. Cela multiplie par deux les chances relatives d'accéder à cette position sociale, tandis qu'avoir des parents sans diplôme réduit presque d'autant cette possibilité. L'effet de la taille de la fratrie disparaît une fois pris en compte le diplôme des individus (tableau 6, modèle 2). Ainsi, la taille de la fratrie influe sur la position sociale des hommes d'abord et avant tout parce qu'elle agit sur le niveau de diplôme obtenu. De même, les effets de la reproduction sociale apparaissent fortement médiatisés par les diplômes que décrochent les individus au sortir des études initiales. Le modèle 2 montre qu'être diplômé du supérieur plutôt que titulaire d'un certificat d'aptitudes

(5) Les fils d'indépendants ne se distinguent pas des fils d'employés ou d'ouvriers dans leurs moindres chances d'ascension sociale. 
professionnelles (CAP) ou d'un brevet d'études professionnelles (BEP) multiplie par 21 les chances d'accéder au statut de cadre plutôt qu'à une position moindre. On voit là un effet du lien très fort en France entre type de diplôme obtenu et emploi occupé.

Ces résultats, relatifs au fait que les fils d'immigrés maghrébins s'en sortent aussi bien que les hommes de la population majoritaire, peuvent paraître contradictoires avec ceux d'études ayant démontré l'existence de discrimination à l'encontre des jeunes issus des immigrations africaines. Toutefois, les modèles ne portent que sur les personnes en emploi (ou ayant occupé un emploi) et qui ont donc franchi la barrière des discriminations à l'emploi. En outre, ils ne tiennent pas compte du type de contrats. Or, la proportion de contrats précaires (y compris dans les positions de cadres) est plus fréquente dans la population issue de l'immigration maghrébine que dans la population majoritaire à niveau de diplôme comparable (Santelli, 2009) (6).

\section{Occuper une positon de cadre : des variations selon l'origine sociale et le sexe}

En moyenne, les filles d'immigrés ont des probabilités moindres que les femmes de la population majoritaire d'être devenues cadres. Mais, si on tient compte de la taille de fratrie, des origines sociales et du capital scolaire des parents (tableau 6,

Tableau 6

Facteurs associés au fait de devenir cadre plutôt que d'accéder à une moindre position sociale (odds ratio)

\begin{tabular}{l} 
\\
\hline Origine migratoire \\
\hline Population majoritaire \\
Enfants d'immigrés venus du Maghreb \\
\hline Origine sociale \\
\hline Ménages d'indépendants \\
Ménages des classes moyennes ou supérieures \\
\hline Ménage des classes populaires \\
\hline Diplôme des parents \\
\hline Aucun diplôme \\
\hline CEP-BEPC \\
CAP-BEP \\
\hline Baccalauréat ou supérieur \\
\hline Nombre de frères et de soeurs \\
\hline Un-e ou moins \\
Deux \\
\hline Trois ou plus \\
\hline Diplôme obtenu \\
\hline Aucun diplôme \\
BEPC \\
CAP-BEP \\
Baccalauréat \\
Diplôme du supérieur \\
\hline
\end{tabular}

\begin{tabular}{|c|c|c|c|c|c|c|c|}
\hline \multicolumn{4}{|c|}{ Hommes } & \multicolumn{4}{|c|}{ Femmes } \\
\hline \multicolumn{2}{|c|}{ Modèle 1} & \multicolumn{2}{|c|}{ Modèle 2} & \multicolumn{2}{|c|}{ Modèle 1} & \multicolumn{2}{|c|}{ Modèle 2} \\
\hline 1,0 & Réf. & 1,0 & Réf. & 1,0 & Réf. & 1,0 & Réf. \\
\hline 1,0 & ns & 0,8 & ns & 2,0 & $* * *$ & 1,7 & *** \\
\hline
\end{tabular}

\begin{tabular}{|l|l|l|l|l|l|l|l|}
\hline 1,2 & $n s$ & 0,8 & $n s$ & 1,6 & $* *$ & 1,3 & $n s$ \\
\hline 2,0 & $* * *$ & 1,4 & $n s$ & 3,2 & $* * *$ & 2,3 & $* * *$ \\
\hline 1,0 & Réf. & 1,0 & Réf. & 1,0 & Réf. & 1,0 & Réf. \\
\hline
\end{tabular}

\begin{tabular}{|c|c|c|c|c|}
\hline 0,6 & $* *$ & 1,0 & $n s$ & 0,6 \\
\hline 0,8 & $n s$ & 1,1 & $n s$ & 0,8 \\
\hline 1,0 & Réf. & 1,0 & Réf. & 1,0 \\
\hline 1,9 & $* * *$ & 1,3 & $n s$ & 1,7 \\
\hline
\end{tabular}

\begin{tabular}{l|l|l|l|l}
$* *$ & 0,9 & $n s$
\end{tabular}

\begin{tabular}{|c|c|c|c|c|c|c|c|}
\hline 1,0 & Réf. & 1,0 & Réf. & 1,0 & Réf. & 1,0 & Réf. \\
\hline 1,2 & $n s$ & 1,1 & $n s$ & 1,0 & $n s$ & 1,0 & $n s$ \\
\hline 0,7 & $* *$ & 0,7 & $n s$ & 0,6 & $* * *$ & 0,7 & $* *$ \\
\hline
\end{tabular}

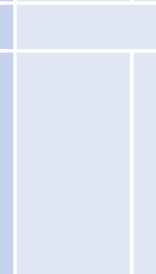

\begin{tabular}{|r|c|}
\hline 0,1 & $* *$ \\
2,0 & $*$ \\
1,0 & Réf. \\
\hline 2,3 & $* * *$ \\
\hline 21,1 & $* * *$ \\
\hline
\end{tabular}

Source: INED-INSEE - Enquête Trajectoires et Origines, 2008

Champ : individus âgés de 30 ans à 50 ans, issus de la population majoritaire ou enfants d'immigres venus du Maghreb.

CEP : certificat d'études primaires; BEPC : brevet d'études du premier cycle du second degré (devenu diplôme national du brevet) ; CAP : certificat d'aptitudes professionnelles; BEP : brevet d'études professionnelles.

Le modèle 1 donne les odds ratio de la régression logistique du fait d'occuper une position sociale subalterne (d'employé-e ou d'ouvrier-ère) et non qualifiée plutôt qu'une position sociale plus avantageuse. II prend en compte l'origine migratoire, l'origine sociale, les diplômes des parents et le nombre de frères et de sœurs; le modèle 2 intègre en plus le diplôme de l'enquêté-e.

" $n s$ » : odds-ratio non significatif; * : significatif au seuil de $10 \% ; * *$ : significatif au seuil de $5 \%$; *** : significatif au seuil de $1 \%$; la mention « Réf. » désigne la modalité de référence.

Lecture du tableau: les filles d'immigrés ont, comparativement aux femmes de la population majoritaire, 0,7 fois moins de risques d'occuper une position sociale subalterne et non qualifiée plutôt qu'une position plus avantageuse, une fois tenu compte de certaines de leurs caractéristiques sociales et familiales (modèle 1).

(6) En outre, ce résultat rejoint les constats d'autres études ayant montré qu'une fois en emploi, les fils d'immigrés venus du Maghreb ne sont plus défavorisés comparativement à la population majoritaire (Dupray et Moullet, 2004). 
modèle 1), le constat est inverse : les filles d'immigrés ont des chances plus élevées que les femmes de la population majoritaire de devenir cadres plutôt que d'occuper une autre position. Toutefois, I'analyse à professions et diplômes identiques des parents ne modifie pas le constat $d^{\prime}$ une forte influence de la taille de la fratrie dans la population féminine, constat qui se révèle donc particulièrement robuste : avoir eu au moins trois frères et sœurs continue, quel que soit le milieu social, de diminuer par deux les chances relatives pour les femmes d'accéder au statut de cadre. Comme pour les hommes, accéder au statut de cadre est d'abord lié au fait d'avoir des parents de position sociale moyenne ou supérieure. L'effet de reproduction sociale apparaît en outre plus prononcé pour elles qu'il ne l'est pour les hommes. Le diplôme acquis par les femmes ne fait pas disparaître le désavantage social que représente le nombre important de frères et de sœurs sur leur position sociale (tableau 6, modèle 2). On voit ici que l'obtention du statut de cadre est plus accessible pour les hommes et que, de surcroît, l'accès à ce statut est moins soumis, en comparaison aux femmes, à l'acquisition d'une certification élevée. Enfin, une fois contrôlées les caractéristiques sociales des familles et des femmes elles-mêmes, il apparaît qu'à taille de fratrie identique, les filles d'immigrés maghrébins réussissent mieux que celles de la population majoritaire.

\section{Occuper une position sociale subalterne et non qualifiée : courant chez les enfants de familles très nombreuses}

Devenir ouvrier ou employé non qualifié plutôt que d'accéder à une meilleure position sociale est, dans la population masculine, plus fréquent chez les fils d'immigrés que chez les hommes de la population majoritaire. Si on compare, à nombre de frères et sœurs identiques, le résultat se maintient mais les écarts sont moindres. Dès que I'on prend en compte la profession et le diplôme des parents (tableau 7, modèle 1 ), ou encore le diplôme obtenu par l'enquêté (tableau 7, modèle 2), la taille de la fratrie perd totalement de sa force explicative. Le fait d'occuper une position subalterne et non qualifiée est avant tout le fait, chez les hommes, d'une forte reproduction sociale de la position sociale des parents. Celle-ci agit essentiellement à travers son influence sur le devenir scolaire : être issu d'un milieu social populaire et être peu diplômé diminue fortement les chances d'acquérir soi-même un diplôme valorisé et favorisent plutôt finalement une position sociale subalterne.
En outre, dans la population masculine, les différences de position sociale selon la taille de la fratrie s'expliquent essentiellement par le fait que les familles très nombreuses sont davantage représentées dans les familles modestes.

Pour les femmes, devenir employée ou ouvrière non qualifiée est, en moyenne, plus fréquent pour les filles d'immigrés que pour les femmes de la population majoritaire. Mais, de nouveau, le constat $s^{\prime}$ inverse une fois prises en compte les caractéristiques familiales et personnelles des femmes : à milieux sociaux (dont la taille des fratries) et diplômes comparables, les filles d'immigrées s'en sortent mieux que les femmes de la population majoritaire (tableau 7). Un nombre élevé de frères et de sœurs paraît plus fortement associé à la probabilité d'occuper une position sociale subalterne et non qualifiée pour les femmes que pour les hommes, y compris lorsque l'on compare, à profession et diplôme des parents identiques (tableau 7, modèle 1), et même lorsque l'on prend en compte le diplôme acquis par les femmes elles-mêmes (tableau 7, modèle 2). Pour les femmes, comme pour les hommes, $\mathrm{n}$ 'avoir aucune qualification est l'élément le plus déterminant du fait d'être employé-e ou ouvrier-ère non qualifié-e. Toutefois, quel que soit le milieu social dont elles sont issues, ou les diplômes qu'elles obtiennent, les femmes sont pénalisées - et non les hommes - d'avoir grandi dans une famille très nombreuse.

La taille de la fratrie constitue un désavantage social - davantage marqué dans la population féminine que masculine - dont l'effet est notamment médiatisé par le diplôme. Ce constat, établi pour la population majoritaire, vaut également pour les enfants d'immigrés. Mais, à caractéristiques familiales (origine sociale, diplôme des parents, taille des fratries) et personnelles (diplômes obtenus) comparables, les différences selon l'origine s'annulent dans la population masculine; elles $\mathrm{s}^{\prime}$ inversent dans la population féminine (7).

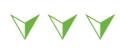

\section{Les hypothèses explicatives}

L'analyse des processus sociaux expliquant le désavantage des enfants de familles nombreuses a été peu traitée. Deux interprétations majeures traversent la littérature dans le domaine : une première met

(7) Ces résultats ne sont pas sans rappeler ceux des études menées sur les scolarités des enfants d'immigrés (Vallet et Caille, 1996 ; Brinbaum et Kieffer, 2005 et 2009). Il faut évidemment être prudent dans ces formulations optimistes, car elles sont fonction d'un système de catégories aux mailles relativement grossières (difficile de faire autrement avec les effectifs de l'enquête) qui néglige par construction le fait que les emplois $d^{\prime}$ « ouvriers» (idem pour les autres catégories) n'ont pas forcément la même structure interne dans les deux populations comparées. 
I'accent sur la dimension culturelle (traditionnelle) pour expliquer la moindre certification scolaire dans ces familles; une autre insiste sur la dimension socio-économique. D. Merllié et O. Monso (2010) analysent, à ce titre, l'impact d'un nombre élevé d'enfants sur les conditions concrètes de la vie scolaire : les conditions matérielles dans lesquelles sont élevés les enfants de familles très nombreuses sont limitées. Les enfants doivent le plus souvent partager leur chambre avec leurs frères et leurs sœurs. Or, disposer de sa propre chambre favoriserait la réussite scolaire (Goux et Maurin, 2002 ; Gouyon, 2004). La suroccupation des logements peut également conduire à "l'extériorisation du temps libre ", avec toutes les conséquences néfastes en termes de déprise éducative pour les parents (Mohammed, 2007). En outre, la faiblesse des ressources économiques des parents est aussi de nature à influencer les choix scolaires des enfants au moment des orientations (par exemple, faire des études courtes pour soulager financièrement les parents). Les travaux de Jean-Pierre Terrail (1984 et 1995) sur la classe ouvrière d'après-guerre fournissent une illustration de la dimension culturelle. Le chercheur a montré que subsistait, à côté des ouvriers qualifiés convertis au mode de vie des classes moyennes, une fraction plus traditionnelle, peu qualifiée et composée de familles (très) nombreuses. La réduction de la fécondité témoignerait, dans les premières, d'un rapport renouvelé et moins fataliste à l'avenir et aux enfants, s'accompagnant de stratégies parentales de scolarisation plus affirmées.

Du point de vue de la dimension économique, les familles immigrées sont plutôt désavantagées : leurs conditions de vie (logements, quartiers, etc.) sont

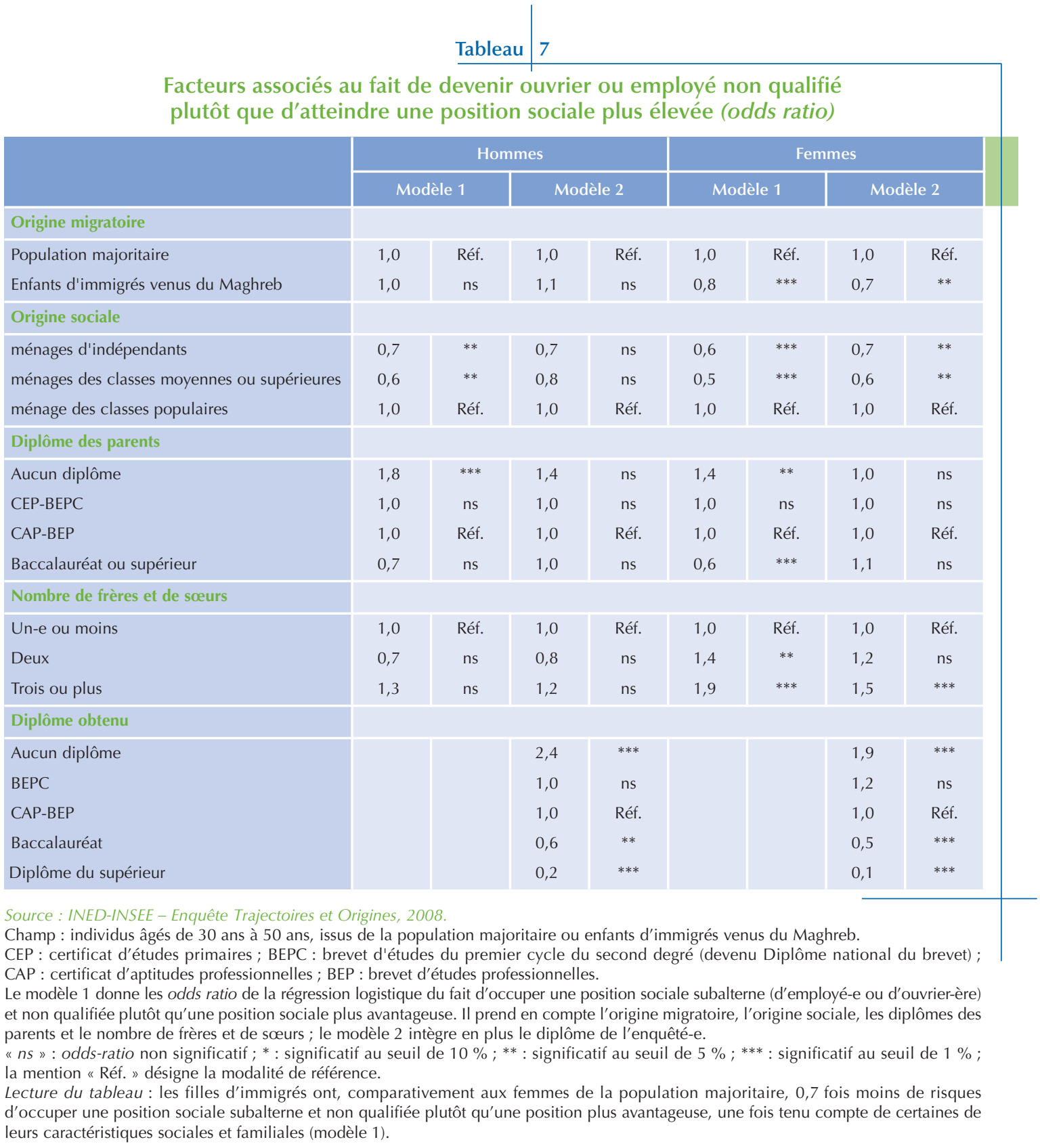


plus précaires que celles de la population majoritaire de dimension comparable (Moguérou et al., 2011). Aussi, I'avantage social des enfants d'immigrés sur ceux de la population majoritaire, que montrent les analyses multivariées, relèverait-il davantage de la dimension dite " culturelle » que de la dimension économique. Pour expliquer le fait que les enfants d'immigrés élevés dans de très grandes familles de milieux populaires s'en sortent mieux que ceux de la population majoritaire à milieux sociaux comparables, plusieurs hypothèses peuvent être avancées. Premièrement, on ne peut réduire la position sociale des familles immigrées au statut professionnel occupé par les parents (le père) en France. En effet, il faudrait pouvoir tenir compte des différences sociales antérieures à la migration (il existe, par exemple, des cas de contre-mobilité) (Santelli, 2001). Deuxièmement, des analyses statistiques ont également montré que, dans les milieux populaires, les aspirations des familles maghrébines en termes de réussite scolaire étaient supérieures à celles de la population majoritaire (Brinbaum et Kieffer, 2005). Ainsi, la forte mobilisation pour la réussite sociale des enfants compenserait, au moins pour une partie d'entre eux, la faiblesse (8) des ressources nécessaires à la réalisation de leur ambition. Cette forte mobilisation est liée au projet migratoire, lui-même étroitement associé à un projet d'ascension sociale expliquant pourquoi bon nombre de familles immigrées - populaires et de très grande taille - se démarquent du milieu traditionnel ouvrier français décrit par Jean-Pierre Terrail : la migration induirait un rapport distinct à I'avenir et aux enfants, s'accompagnant de mobilisations scolaires des parents plus affirmées. Troisièmement, l'entraide entre frères et sœurs est plus fréquente dans les familles immigrées de (très) grande taille que dans celles non immigrées de mêmes dimensions (Moguérou et al., 2011). L'enquête qualitative réalisée sur les fratries (Moguérou et Santelli, 2012) montre que c'est là une ressource spécifique de ces familles, et qu'elle $\mathrm{s}^{\prime}$ exprime de trois manières principalement :

- I'entrée des aînés sur le marché du travail procure des ressources financières supplémentaires dont bénéficient les cadets, notamment pour la poursuite d'études; ils mettent à la disposition du ménage tout ou une grande partie de leurs revenus ; - les cadets disposent des connaissances acquises par leurs aînés, concernant les filières scolaires notamment. Les aînés servent en effet, de modèles, en montrant la trajectoire à suivre ou en mettant à profit les enseignements de leurs erreurs pour guider leurs plus jeunes frères et sœurs ;

- au quotidien, les aînés peuvent également se substituer aux parents en apportant une aide scolaire, et ce d'autant plus qu'ils incarnent une réussite scolaire (aide à la réalisation des devoirs, encouragement, surveillance du carnet de notes, etc.).

Ces dynamiques familiales à l'œuvre expliqueraient comment des enfants de familles maghrébines parviennent à obtenir une position sociale élevée quand, du fait des conditions sociales et économiques de leurs parents, cette mobilité paraissait hautement improbable.

Dernière hypothèse explicative pour tenter de comprendre pourquoi la comparaison entre individus de conditions comparables (taille de la fratrie et milieu social) est à l'avantage des enfants d'immigrés maghrébins : dans ces familles, avoir un grand nombre d'enfants constitue la norme, elles savent «faire avec », ce qui n'est pas le cas dans la population majoritaire où les familles très nombreuses sont " hors norme » : la famille à deux ou trois enfants correspond, aujourd'hui, à une préférence largement dominante, que l'on s'intéresse au nombre idéal d'enfants ou aux souhaits de fécondité (Régnier-Loilier, 2006). Cela revient à dire que, dans les deux populations comparées, la dimension de la fratrie n'a pas toujours le même sens.

Enfin, il ressort de l'analyse qu'il existe de fortes disparités entre les hommes et les femmes [résultat qui rejoint l'étude de D. Merllié et Monso (2007)]. Pour les hommes, l'effet «taille de la fratrie » est annulé quand on introduit la variable «diplôme obtenu ", alors qu'il persiste pour les femmes. Ainsi, le fait d'avoir grandi dans une famille très nombreuse joue sur l'obtention du diplôme, pour les hommes comme pour les femmes, mais, pour ces dernières, il demeure un effet persistant " au-delà du diplôme ». Ces écarts s'expliqueraient à la fois par ce qui se passe en amont, durant le parcours scolaire, au cours de la socialisation familiale et juvénile, puis en aval, lors de l'accès au marché du travail et dans le déroulement de la carrière. À ces différentes étapes, les hommes et les femmes ont des parcours distincts et des expériences différenciées. Alors que dans les familles populaires les jeunes femmes sont davantage pénalisées (comparativement aux hommes) en raison de la prise en charge des tâches domestiques, à l'âge adulte, elles ont aussi plus souvent la charge de leurs cadets, des parents vieillissants, une moindre implication dans leur vie professionnelle en raison de comportements plus statutaires (un rapport au travail différencié dans lequel l'homme est le principal pourvoyeur de revenus). Ainsi, les rôles sociaux de sexe jouent en défaveur de l'ascension sociale des femmes, en particulier dans les familles nombreuses. De plus, la parentalité n'a pas non plus la même signification selon les milieux sociaux.

(8) Liée à leur manque d'informations sur le système scolaire et à la faiblesse de leurs revenus. 
Dans les milieux ouvriers, accéder rapidement au statut de parent est socialement valorisé, à la fois pour les femmes et pour les hommes (Schwartz, 1990). Par ailleurs, Arnaud Régnier-Loilier (2006) a montré l'existence d'un effet de la taille de la famille d'origine sur les comportements féconds (9): plus on est issu d'une fratrie étendue, plus on souhaite avoir d'enfants et plus on en a. Or, le fait d'avoir des enfants tôt et d'en avoir beaucoup est le plus souvent un frein dans les carrières des femmes.

De manière générale, les femmes de la population majoritaire semblent reproduire les rôles familiaux, ce qui tendrait in fine à les pénaliser sur le marché du travail et à expliquer les écarts observés avec les femmes d'origine maghrébine. Ces dernières, en raison de leur histoire familiale, ressentiraient un profond sentiment d'injustice et auraient à cœur de ne pas reproduire la situation de leur mère. À la différence de leurs frères, les filles ont consacré nettement plus de temps aux travaux domestiques (10). Les filles ont également été plus étroitement surveillées quant à leurs sorties, et elles ont passé plus de temps à la maison que les garçons. Si cette situation peut être favorable à une plus grande implication dans la réalisation du travail scolaire, elle a pu aussi provoquer une volonté d'émancipation que ces filles ont tenté d'obtenir par le biais de leur réussite scolaire puis professionnelle. S'extraire de leur condition sociale et familiale a pu également être soutenu et encouragé par leur mère. Les inégalités subies par ces femmes, filles et mères, peuvent expliquer leur plus grande volonté à assurer leur avenir social. Pour cette raison également, les filles d'immigrés sont moins disposées à reproduire les comportements de fécondité de leurs parents. Ainsi, les filles d'immigrés seraient enclines, plus fréquemment que les femmes des milieux populaires ayant grandi dans de très grandes familles, à s'opposer à la perpétuation des rôles sociaux de sexe. Plus affranchies, ou pour le moins plus soucieuses de la renégociation de ces normes familiales, elles bénéficieraient aussi du soutien des institutions, et en premier lieu de l'école, pour se distancier des modèles parentaux transmis. Si l'effet «taille de la fratrie » est persistant dans la population féminine, il apparaît donc moins discriminant pour les filles d'immigrés que pour celles de la population majoritaire.

\footnotetext{
9) D'anciens travaux menés en France par Guy Desplanques (1987), dans le prolongement de ceux engagés par Jean-Claude Deville (1979), ont démontré l'existence d'une liaison positive entre la taille de la fratrie d'origine et la descendance finale : plus on a de frères et de sœurs, et plus on a soi-même d'enfants, en moyenne. Mais ces résultats avaient été nuancés : "I'homogamie explique une partie de cette hérédité apparente. On se marie souvent dans le groupe social d'origine ou dans la même région. Ce faisant, on a tendance à reproduire les comportements traditionnels du milieu social ou les habitudes locales» (Desplanques, 1987 - cité par Régnier-Loilier, 2006:206).

(10) Les entretiens (Moguérou et Santelli, 2012) témoignent de l'écart de traitement entre les filles et les garçons dans une grande majorité de familles immigrées concernant l'implication dans les tâches domestiques.
} 


\section{Références $\mid$ bibliographiques}

- Aeberhardt R. et Pouget J., 2010, National origin differences in wages and hierarchical positions: Evidence on french full-time male workers from a matched employer-employee dataset, Annals of Economics and Statistics, n 99/100:117-139.

- Baraton M., 2006, " De la difficulté à devenir cadre par promotion », INSEE Première, n 1062.

- Beauchemin C., Hamel C. et Simon P., 2010, "Trajectoires et Origines. Enquête sur la diversité des populations en France », Documents de travail, Institut national d'études démographiques, n 168.

- Borrel C. et Thave S., 1989, Familles nombreuses : $22 \%$ des familles, $40 \%$ des enfants, Économie et Statistique, vol. 224, $n^{\circ} 1: 51-55$

- Bouhmadi R. et Giret J.-F., 2005, Une analyse économétrique des disparités d'accès à l'emploi et de rémunérations entre jeunes d'origine française et jeunes issus de l'immigration, Revue économique, vol. 56, n 3:625-636.

- Bresard M., 1950, Mobilité sociale et dimension de la famille, Population, vol. 5, n 3:533-566

- Brinbaum Y. et Kieffer A., 2009, Les scolarités des enfants d'immigrés de la sixième au baccalauréat : différenciation et polarisation des parcours, Population, vol. 64, n 3:561-610.

- Brinbaum Y. et Kieffer A., 2005, D’une génération à l'autre, les aspirations éducatives des familles immigrées : ambition et persévérance, Éducation et formations, $n^{\circ} 72: 53-75$.

- Desplanques G., 1987, « Calendrier des familles », Données sociales, INSEE:477-481.

- Deville J.-C., 1979, La fécondité serait-elle héréditaire ?, Economie et Statistique, n 116:3-11.

- Dupray A. et Moullet S., 2004, "L'insertion des jeunes d'origine maghrébine en France. Des différences plus marquées dans l'accès à l'emploi qu'en matière salariale», collection Net.Doc, Centre d'études et de recherches sur les qualifications, $n^{\circ} 6$.

- Frickey A., Murdoch J. et Primon J.-L., 2004, «Les débuts dans la vie active des jeunes issus de l'immigration après des études supérieures", Notes emploi-formation (Nef), Centre d'études et de recherches sur les qualifications, nº 9.

- Gaymu J. et Parant A., 1996, Les débuts dans la vie active des jeunes immigrés et des jeunes d'origine étrangère, Espace populations sociétés, $\mathrm{n}^{\circ}$ 2/3:439-455.

- Goux D. et Maurin E., 2002, "Surpeuplement du logement et retard scolaire des enfants », Données sociales : la société française, INSEE:455-459.

- Gouyon M., 2004, "L'aide aux devoirs apportée par les parents », INSEE Première, n 996.

- Institut national de la statistique et des études économiques (INSEE), 2005, "Les immigrés en France », collection Références, Paris.

- Merllié D. et Monso O., 2010, "Mobilité sociale et dimension de la famille : une question oblitérée », http://www.melissa.ens-cachan.fr/IMG/pdf/Merllie_Monso_2010-4.pdf

- Merllié D. et Monso O., 2007, "La destinée sociale varie avec le nombre de frères et sœurs », France Portrait Social, INSEE:135-153.

- Meron M. et Minni C., 1995, Des études à I'emploi : plus tard et plus difficilement qu'il y a vingt ans, Economie et Statistique, $\mathrm{n}^{\circ}$ 283-284:9-31.

- Meurs D., Pailhé A. et Simon P., 2006, Persistance des inégalités entre générations liées à l'immigration : l'accès à l'emploi des immigrés et de leurs descendants en France, Population, vol. 5-6, n 61:763-802.

- Moguérou L. et Santelli E., 2012, Parcours scolaires réussis d'enfants d'immigrés issus de familles très nombreuses, Informations sociales, $\mathrm{n}^{\circ} 173: 84-92$.

- Moguérou L., Hamel C., Primon J.-L., Santelli E. et Charruault A., 2011, « La taille des familles et le devenir scolaire et socioprofessionnel des enfants d'immigrés ", rapport de recherche pour la Caisse nationale des Allocations familiales, non publié.

- Mohammed M., 2007, Fratries, collatéraux et bandes de jeunes, in Les bandes de jeunes. Des blousons noirs à nos jours (sous la dir. de Mucchielli L. et Mohammed M.), Paris, La Découverte:97-122.

- Monso O., 2006, "Changer de groupe social en cours de carrière », INSEE Première, n 1112.

- Okba M., 2012, "Métiers des pères et des descendants d'immigrés : une mobilité sociale davantage liée à l'origine sociale qu'à I'origine ethnique ", Dares Analyses, $\mathrm{n}^{\circ} 58$.

- Régnier-Loilier A., 2006, L'influence de la taille de la fratrie sur le nombre souhaité d'enfants à différents moments de la vie. L'exemple de la France, Population, vol. 61, n 3:193-223.

- Santelli E., 2001, La mobilité sociale dans l'immigration. Itinéraires de réussite des enfants d'origine algérienne, Toulouse, Presses Universitaires du Mirail, collection Socio-logiques.

- Santelli E., 2009, Ne pas se sentir discriminé : une analyse des parcours atypiques au croisement d'une analyse micro et macro, Sociologie et Sociétés, vol. 41, n 1:263-290.

- Schwartz O., 1990, Le monde privé des ouvriers. Hommes et femmes du Nord, Paris, PUF.

- Silberman R. et Fournier I., 2006, Les secondes générations sur le marché du travail en France : une pénalité ethnique ancrée dans le temps, Revue française de sociologie, vol. 47, $\mathrm{n}^{\circ}$ 2:243-292.

- Simon P., 2008, Les statistiques, les sciences sociales françaises et les rapports sociaux ethniques et de race, Revue française de sociologie, vol. 1, $n^{\circ}$ 49:153-162.

- Tabard N., 1984, Mobilité sociale, fratrie et descendance, Consommation, XXXI, n 3:19-50.

- Tabard N., 1986, Mobilité sociale entre les générations et taille de la fratrie, Le groupe familial, n 111:54-63.

- Terrail J.-P., 1984, Familles ouvrières, école, destin social, Revue française de sociologie, XXV, n 3:421-436.

- Terrail J.-P., 1995, La dynamique des générations : activité individuelle et changement social (1968/1993), Paris, L’Harmattan.

- Tribalat M., 1995, Faire France, Paris, La Découverte.

- Vallet L.-A. et Caille J.-P., 1996, "Les élèves étrangers ou issus de l'immigration dans l'école et le collège français » Les Dossiers d'éducation et formation, $n^{\circ} 67$. 